\title{
Obesity, Echocardiographic Changes and Framingham Risk Score in the Spectrum of Gout: A Cross-Sectional Study
}

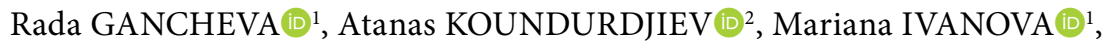 \\ Todor KUNDURZHIEV (1) ${ }^{3}$, Zlatimir KOLAROV (1) ${ }^{1}$ \\ ${ }^{1}$ Department of Internal Medicine, Clinic of Rheumatology, Medical University, University Hospital St. Iv. Rilski, Sofia, Bulgaria \\ ${ }^{2}$ Department of Internal Medicine, Clinic of Nephrology, Medical University, University Hospital St. Ivan Rilski, Sofia, Bulgaria \\ ${ }^{3}$ Medical University, Faculty of Public Health, Sofia, Bulgaria
}

\begin{abstract}
Objectives: This study aims to establish cardiovascular risk in obese and non-obese patients in stages of gout by using Framingham risk score (FRS) and transthoracic echocardiography.

Patients and methods: This single-center cross-sectional study encompassed 201 patients (160 males, 41 females; mean age $56.9 \pm 13$ years; range 20 to 89 years) including 52 asymptomatic hyperuricemia, 86 gouty arthritis without tophi, and 63 gouty tophi patients. body mass index (BMI) and FRS were calculated. Left atrium (LA), interventricular septum, posterior wall (PW) of the left ventricle, fractional shortening (FS), mitral annular systolic velocity $\left(S^{\prime}\right)$, mitral annular early diastolic velocity $\left(E^{\prime}\right)$ and transmitral to mitral annular early diastolic velocity ratio $\left(E / E^{\prime}\right)$ were measured. Data were analyzed by Kolmogorov-Smirnov test, Shapiro-Wilk test, t-test, Mann-Whitney $U$ test, analysis of variance test and multiple linear regression models.

Results: There was no significant difference in FRS, FS, $S^{\prime}, E^{\prime}$ and $E / E^{\prime}$ between obese and non-obese patients with asymptomatic hyperuricemia, gouty arthritis without tophi or gouty tophi. Obese patients in the three disease gradations had larger $L A(p=0.007, p=0.004, p=0.039)$ and thicker PW $(p=0.002, p=0.037, p=0.007)$. Increased BMI independently predicted the thickening of the $P W$ in asymptomatic hyperuricemia $\left(R^{2}=0.319\right)$, gouty arthritis without tophi $\left(R^{2}=0.093\right)$ and gouty tophi $\left(R^{2}=0.068\right)$.

Conclusion: Despite the lack of difference in FRS and functional systolic and diastolic parameters between obese and non-obese patients in the spectrum of gout, morphological heart changes were more pronounced in obese patients. In gouty tophi, it is possible that higher urate load together with chronic inflammation contribute for the alterations, as obesity worsens them.

Keywords: Cardiovascular risk, gout stages, obesity.
\end{abstract}

The rates of obesity are increasing worldwide. ${ }^{1}$ Its prevalence has increased dramatically not only in adults, but also in children and adolescents in developed and developing countries. It has turned out to be a major health concern. ${ }^{2}$ Given that, obesity is associated with increased morbidity and mortality rates. ${ }^{3}$ Increased adiposity is linked to hyperuricemia. ${ }^{4}$ Higher body mass index (BMI) and weight gain contribute for gout in males. ${ }^{5}$ It is considered that the increased prevalence of gout is partly due to the epidemic of obesity. ${ }^{6}$ Studies have proven a strong connection between obesity, hyperuricemia and higher risk of gout., ${ }^{4,8}$ Even more, it has been suggested that obesity may be a factor linking hyperuricemia, hypertension, dyslipidemia and atherosclerosis. ${ }^{5}$

Obesity is a well-recognized risk factor for cardiovascular complications. ${ }^{3}$ In the last decade, a great number of studies have made evident

Received: July 28, 2018 Accepted: August 02, 2018 Published online: January 28, 2019

Correspondence: Rada Gancheva, MD. Department of Internal Medicine, Clinic of Rheumatology, Medical University, University Hospital St. Iv. Rilski, 1612 Sofia, Bulgaria. Tel: 00359889593183 e-mail: rada_ga@mail.bg 
the importance of hyperuricemia and gout in cardiovascular pathology. Some authors even demonstrated that chronic tophaceous gout is a qualitatively new disease characterized by high cardiovascular risk. $^{9-11}$ However, obesity and gout are frequently combined, which hinders the assessment of the risk. This gave us reason to determine the contribution of obesity to cardiovascular risk and development of heart impairments in the individual stages of gout by using the classic and one of the most commonly applied scoring system in everyday clinical practice, namely the Framingham risk score (FRS) and transthoracic echocardiography. Morphological and functional parameters of systole and diastole of the heart were measured with the latter imaging modality. We took into consideration the earlier findings indicating that left atrium (LA) size reflects the severity of left ventricular diastolic dysfunction for a prolonged period of time. ${ }^{10,12}$ Mitral annular early diastolic velocity $\left(E^{\prime}\right)$ is a sensitive indicator of diastolic dysfunction. Unlike the left atrium size, the measurement of $E^{\prime}$ cannot judge the duration of diastolic dysfunction. ${ }^{13,14}$ Interventricular septum (IVS) thickness and thickness of the posterior wall $(\mathrm{PW})$ of the left ventricle give a notion for the presence of left ventricular hypertrophy, ${ }^{15}$ resulting in diastolic dysfunction. Transmitral to mitral annular early diastolic velocity ratio (E/E') has been shown to be useful to assess left ventricular filling pressure at a fixed point of time.13,16 Fractional shortening (FS) and mitral annular systolic velocity (S') are common parameters of systolic function of the left ventricle. ${ }^{17,18}$ We conducted our study bearing in mind that obesity leads to hemodynamic overload with subsequent expanded intravascular remodeling. ${ }^{19}$ Therefore, in this study, we aimed to establish cardiovascular risk in obese and nonobese patients in stages of gout by using FRS and transthoracic echocardiography.

\section{PATIENTS AND METHODS}

This single-center cross-sectional study encompassed 201 age-matched patients (160 males, 41 females; mean age 56.9 \pm 13 years; range 20 to 89 years) divided into three groups: 52 asymptomatic hyperuricemia, 86 gouty arthritis without tophi and 63 gouty tophi patients
(Table 1). We analyzed consecutive patients hospitalized in University Rheumatology Clinic of "St. Ivan Rilski" Hospital in Sofia between September 2013 and May 2016. Some of the gout patients were hospitalized in view of the diagnosis, other for management of a continuous attack or choice of therapy in those with chronic tophaceous gout. Asymptomatic hyperuricemia individuals attended the outpatient clinic. They had no inflammatory arthritis or other autoimmune disease and their serum uric acid level was above the upper limit of normal with no history of gout flare. No imaging method was performed in this group for detection of silent monosodium urate crystals. Gout patients fulfilled the 2015 American College of Rheumatology and European League Against Rheumatism classification criteria, ${ }^{20}$ as gouty tophi were described in individuals with subcutaneous tophi on physical examination. Synovial fluid analyses for detection of monosodium urate crystals and ultrasound examination for double contour sign were performed when appropriate. The study protocol was approved by the Medical University of Sofia Ethics Committee (20 May 2013, protocol No: 15). A written informed consent was obtained from each patient. The study was conducted in accordance with the principles of the Declaration of Helsinki.

A rheumatologist reviewed the medical documentation and performed physical examination of the subjects. Obesity was defined as BMI $>30 \mathrm{~kg} / \mathrm{m}^{2} .{ }^{21}$ The FRS was calculated by an internet calculator. FRS assesses the individual's 10-year risk for developing cardiovascular disease via seven items concerning sex, age, total cholesterol, high density lipoprotein cholesterol, smoking, the value of systolic blood pressure, and treated and untreated arterial blood pressure. According to this scoring system, individuals with low risk have $10 \%$ or less coronary heart disease risk at 10 years, those with intermediate risk have 10-20\% and those with high risk have $20 \%$ or more risk..$^{22,23}$ Other clinical and laboratory assessments included current smoking status; arterial hypertension defined as systolic blood pressure $\geq 140 \mathrm{mmHg}$, diastolic blood pressure $\geq 90 \mathrm{mmHg}$ or use of antihypertensive medication; diabetes mellitus, based on physician's diagnosis and/or documented use of insulin and/or oral hypoglycemic agents; 
dyslipidemia, based on elevated fasting lipid levels or low high density lipoprotein cholesterol and/or documented use of lipid-lowering agents; reduced glomerular filtration rate taken at estimated glomerular filtration rate (eGFR) $<90 \mathrm{~mL} /$ minute calculated by the CockroftGault formula, chronic kidney failure (CKF) as accepted by Kidney Disease Outcomes Quality Initiative (K/DOQI) 2002 guidelines ${ }^{24}$ and other hematological and biochemistry parameters including the inflammatory biomarker $\mathrm{C}$-reactive protein (CRP). Hyperuricemia was adopted when serum level of uric acid was higher than $6.0 \mathrm{mg} / \mathrm{dL}$ (356.9 $\mu \mathrm{mol} / \mathrm{L})$ for females and higher than $6.8 \mathrm{mg} / \mathrm{dL}$ (404.5 $\mu \mathrm{mol} / \mathrm{L})$ for males. A single measurement of the laboratory parameters was taken for the analyses. Patients were defined as having suffered a cardiovascular event if they had coronary artery disease, cerebrovascular disease and/or peripheral artery disease. $^{25}$ Exclusion criteria were a history of malignancy, exacerbated cardiac or renal failure, dilated or hypertrophic cardiomyopathy, severe valvulopathy, atrial fibrillation, arterial blood pressure $>140 / 90 \mathrm{mmHg}$ and heart rate $<50$ bpm or $>90$ bpm during the ultrasonographic measurement.

Transthoracic echocardiography was performed by an experienced certified ultrasonographer, who was unaware with the cases' clinical data. Ultrasound examinations were conducted on ALOKA-SSD-4000 (ALOKA CO LTD 6-22 I Mure Mitaka-shi, Tokyo, Japan) between June 2013 and November 2015. Measurements were performed on Philips HD11 between 20 November 2015 and May 2016. Software of both devices had comparable characteristics. Examinations were carried out according to the recommendations of the American Society of Echocardiography with $2.5 \mathrm{MHz}$ transducer working with pulsed Doppler frequency of $2.5 \mathrm{MHz}$. A minimum of three cardiac cycles were recorded, analyzed and averaged. IVS thickness and thickness of the PW of the left ventricle were determined at end-diastolic phase with two-dimentionally-guided M-mode. From the parasternal long axis view, uniaxial anterior-posterior dimension of the LA was assessed. FS was estimated by the method of Teicholz. When applying pulse-Doppler for measurement of transmitral annular early diastolic velocity (E), the probe volume of $2 \mathrm{~mm}$ was placed at the apex of the mitral valve in a four-chamber view. ${ }^{18,26} S^{\prime}$ and $E^{\prime}$ velocities were recorded with tissue Doppler imaging (TDI). During TDI, the probe volume of $2 \mathrm{~mm}$ was placed on the lateral wall at the level of mitral valve annulus in order to avoid the influence of the right ventricle. ${ }^{27}$ The $\mathrm{E} / \mathrm{E}^{\prime}$ was obtained automatically from the computer software.

\section{Statistical analysis}

Data were analyzed using the Statistical Package for the Social Sciences version 13.0 (SPSS Inc., Chicago, IL, USA). Means with standard deviations (SDs) and percentages were calculated to describe the clinical and echocardiographic characteristics of the patients. The distribution of data was checked with Kolmogorov-Smirnov and Shapiro-Wilk tests. Intergroup comparisons were performed by analysis of variance, Kruskal-Wallis, post hoc Tukey, t-test and Mann-Whitney U tests. Proportional differences were tested using the chi-square $\left(\chi^{2}\right)$, Fisher's exact tests and z-test with Bonferroni adjustment. To assess the impact of BMI on PW in the stages of gout, we performed a multiple linear regression analysis, adjusted for age, with an independent factor of BMI and dependent variable of PW of the left ventricle. Results were expressed as regression coefficients.

\section{RESULTS}

The three examined groups were agematched $(p=0.290)$. Subjects with acute flare were equally divided in both gout groups $(p=0.110)$. However, gouty tophi patients had the highest values of CRP $(p=0.001)$ in intergroup comparison. Serum concentration of uric acid was similar in both gout groups $(p=0.097)$. Expectedly, it was higher in chronic tophaceous gout than in asymptomatic hyperuricemia subjects $(p=0.006)$. In the three gradations of the disease process, the means of eGFR did not differ significantly $(p=0.075)$, but the percentage of CKF patients was higher in gouty tophi compared to gouty arthritis without tophi $(p=0.015)$. The frequency of the cases with CKF in asymptomatic hyperuricemia group was comparable to this in gouty tophi $(p=0.136)$. 


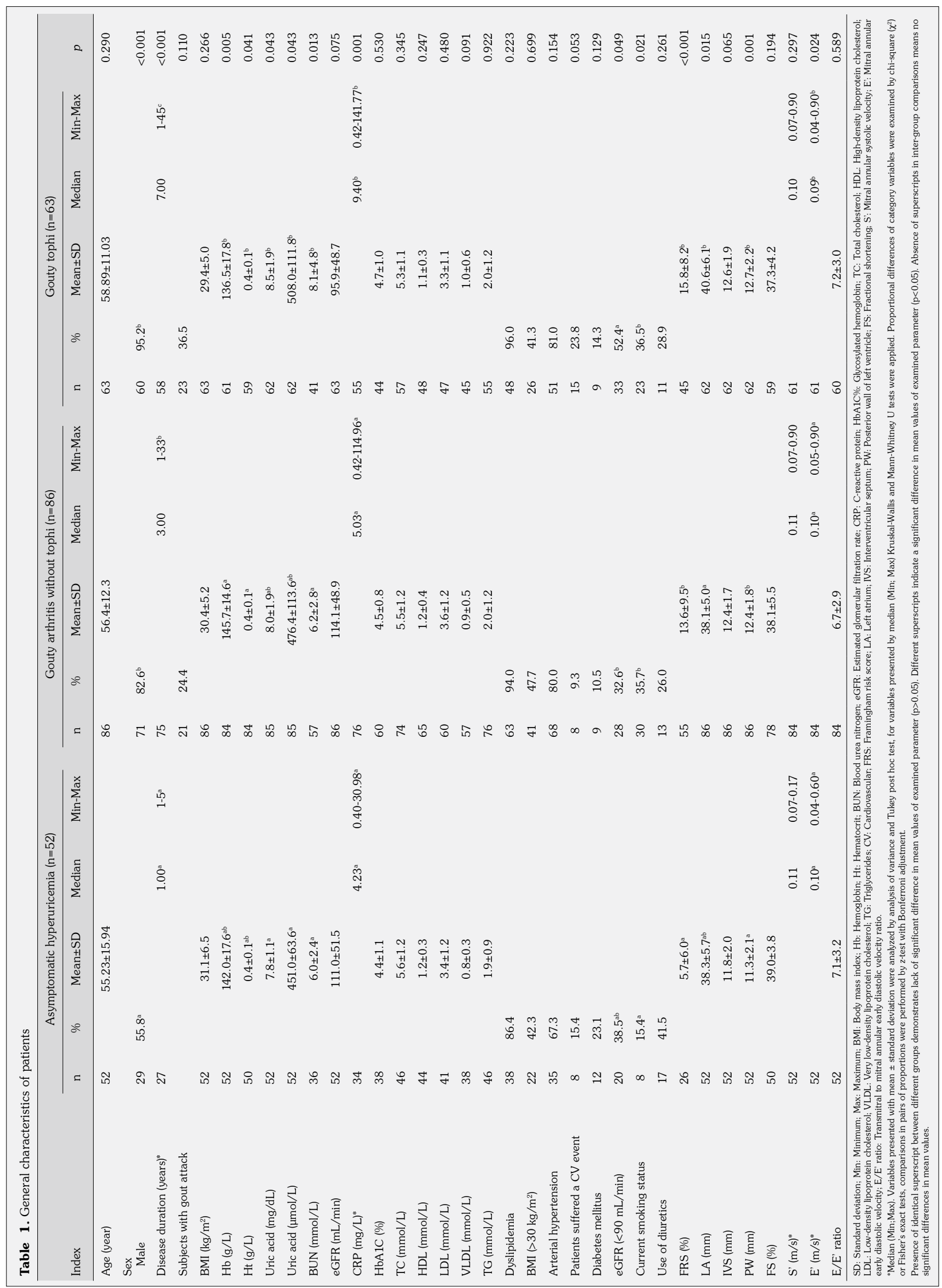


In the spectrum of gout BMI $(p=0.266)$, prevalence of obesity $(p=0.699)$, dyslipidemia $(p=0.223)$, arterial hypertension $(p=0.154)$, previous cardiovascular event $(p=0.053)$, diabetes mellitus $(p=0.129)$ and the proportion of patients treated with diuretics $(p=0.261)$ was similar. As opposed to asymptomatic hyperuricemia, the distribution of smokers was higher in gout $(p=0.021)$. According to the FRS, cardiovascular risk was higher in gout than in asymptomatic hyperuricemia subjects. Gouty arthritis without tophi (mean \pm SD; $13.62 \pm 9.48 \%$ ) and gouty tophi (mean \pm SD; 15.79 $\pm 8.24 \%$ ) had an intermediate risk, while those with asymptomatic hyperuricemia were scored with a low risk (mean $\pm S D ; 5.69 \pm 6.03 \%$ ). Analyzing echocardiographic changes, we did not observe any significant deviation in the thickness of the IVS $(p=0.065), F S(p=0.194), S^{\prime}(p=0.297)$ or $E / E^{\prime}$ ratio $(p=0.589)$ in the stages of gout. LA was larger in patients with gouty tophi than in those with gouty arthritis without tophi $(p=0.017)$, but no significant difference in its size was estimated between tophi and asymptomatic hyperuricemia patients $(p=0.067)$. Unlike the latter, $\mathrm{PW}$ of the left ventricle was thicker in both gout groups $(p=0.001)$. The mean values of $E^{\prime}$ were significantly the lowest in gouty tophi $(p=0.024)$ (Table 1).

In the group of asymptomatic hyperuricemia, 22 of the patients were obese and 30 were nonobese; in the group of gouty arthritis without tophi, 41 were obese and 45 were non-obese, while in the group with tophi, the number of obese patients was 26 and of non-obese 37. We did not estimate any significant difference in the serum concentration of uric acid, distribution of dyslipidemia, arterial hypertension, previous cardiovascular event, smoking or use of diuretics between obese and non-obese in the three examined groups. In tophaceous gout, the distribution of diabetes mellitus was higher in obese subjects than in non-obese. In the spectrum of disease, only a few of the patients with obesity had an abnormally low eGFR (Table 2).

The scores obtained by using the FRS were comparable between obese and non-obese in asymptomatic hyperuricemia, gouty arthritis without tophi and gouty tophi $(7.54 \pm 7.91 \%$ vs. $4.34 \pm 3.96 \%, \quad p=0.187 ; \quad 12.23 \pm 8.13 \%$ vs. $15.28 \pm 10.81 \%, \quad p=0.238 ; 15.81 \pm 8.39 \%$

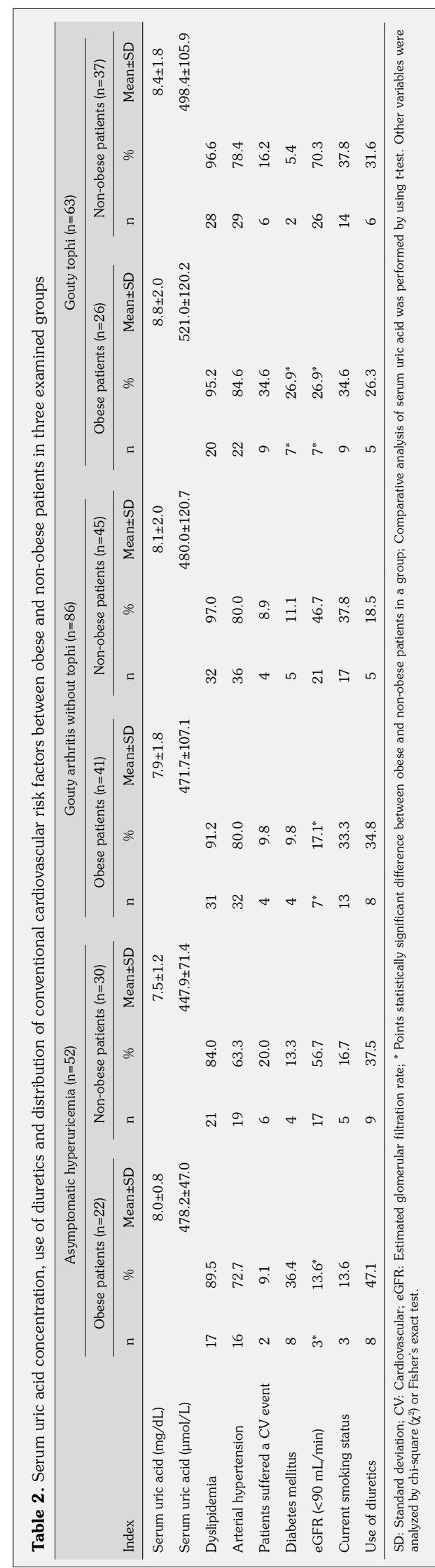


vs. $15.77 \pm 8.30 \%, \quad p=0.990$, respectively) (Figure 1a). From earlier to later stages of the disease in obese and non-obese, functional parameters of systole and diastole had similar mean values (Figure $1 \mathrm{~b}-\mathrm{e}$ ). Obese subjects with asymptomatic hyperuricemia, gouty arthritis without tophi and chronic tophaceous gout had larger LA compared to non-obese $(40.68 \pm 5.22 \mathrm{~mm}$ vs. $36.50 \pm 5.37 \mathrm{~mm}, \mathrm{p}=0.007$; $39.66 \pm 4.36 \mathrm{~mm}$ vs. $36.60 \pm 5.12 \mathrm{~mm}, \mathrm{p}=0.004$; $42.45 \pm 5.96$ vs. $39.25 \pm 5.84 \mathrm{~mm}, p=0.039$, respectively) (Figure 2a). Obese subjects in the group of asymptomatic hyperuricemia had thicker IVS. The latter did not demonstrate any difference between obese and non-obese patients in gouty arthritis without tophi and gouty tophi groups (Figure 2b). Regarding the $\mathrm{PW}$ of the left ventricle, it was thicker in obese individuals in the stages of gout

(a)

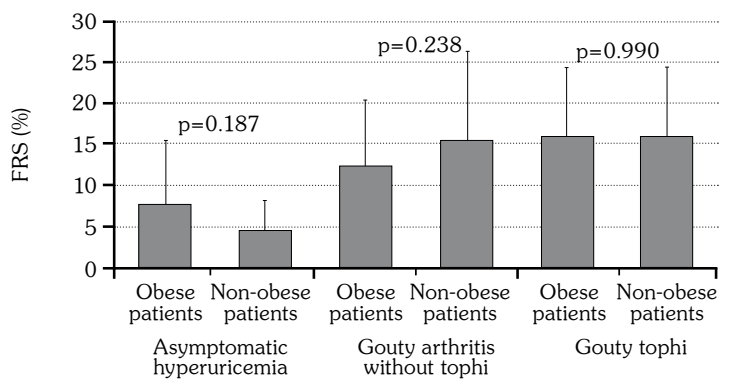

(c)

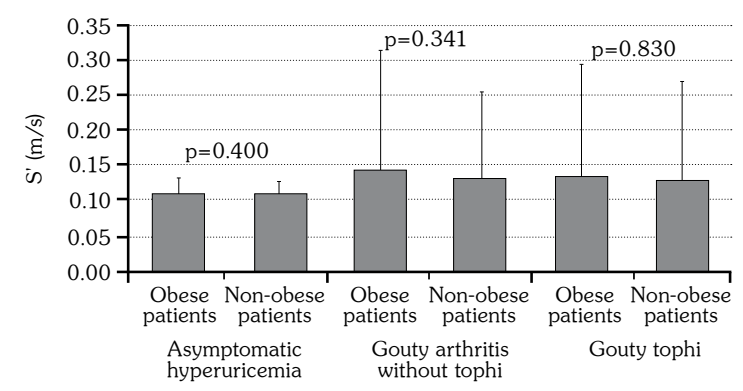

(e)

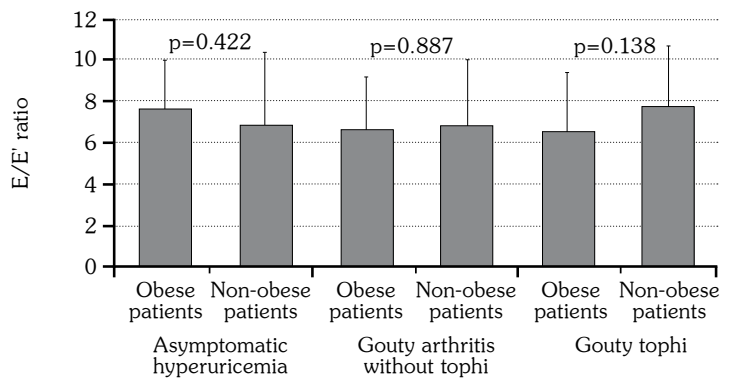

$(12.41 \pm 1.66 \mathrm{~mm}$ vs. $10.48 \pm 2.03 \mathrm{~mm}, \mathrm{p}=0.002$ $12.71 \pm 1.67 \mathrm{~mm}$ vs. $12.05 \pm 1.89 \mathrm{~mm}, \mathrm{p}=0.037$; $13.50 \pm 2.46 \mathrm{~mm}$ vs. $12.13 \pm 1.77 \mathrm{~mm}, \mathrm{p}=0.007$, respectively) (Figure 2c).

Finally, we analyzed the influence of BMI on the thickness of the left ventricular posterior wall. A multiple linear regression analysis adjusted for age was conducted, through which we established that the increase of BMI independently predicted the thickening of the $\mathrm{PW}$ in asymptomatic hyperuricemia $\left(\mathrm{R}^{2}=0.319\right)$, gouty arthritis without tophi $\left(R^{2}=0.093\right)$ and gouty tophi $\left(R^{2}=0.068\right)$ (Table 3).

\section{DISCUSSION}

The present study reveals that obese subjects in the spectrum of gout have more pronounced

(b)

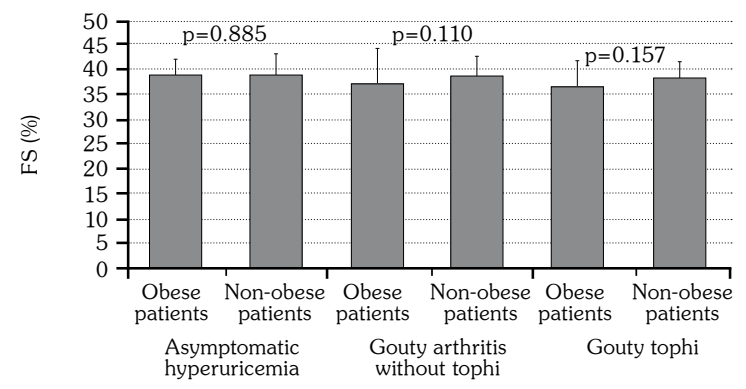

(d)

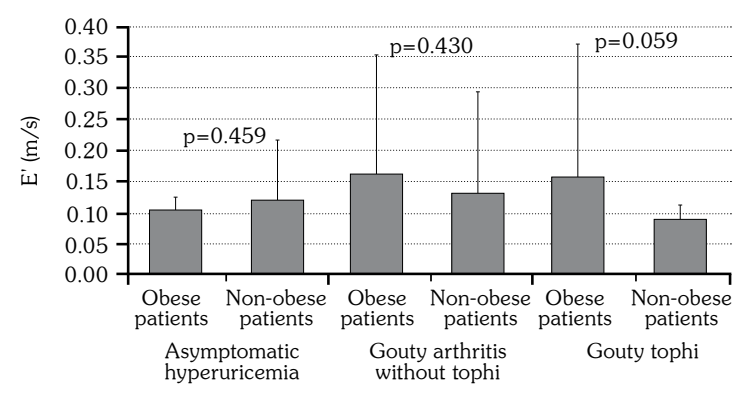

Figure 1. Mean and standard deviation of (a) Framingham risk score, (b) fractional shortening, (c) mitral annular systolic velocity, (d) mitral annular early diastolic velocity and (e) transmitral to mitral annular early diastolic velocity ratio. FRS: Framingham risk score; FS: Fractional shortening; S: Mitral annular systolic velocity; E`: Mitral annular early diastolic velocity; E/E`: Transmitral to mitral annular early diastolic velocity ratio. 
(a)

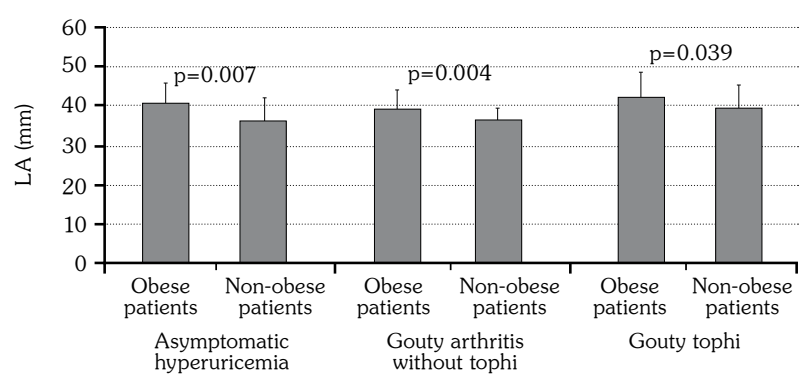

(b)

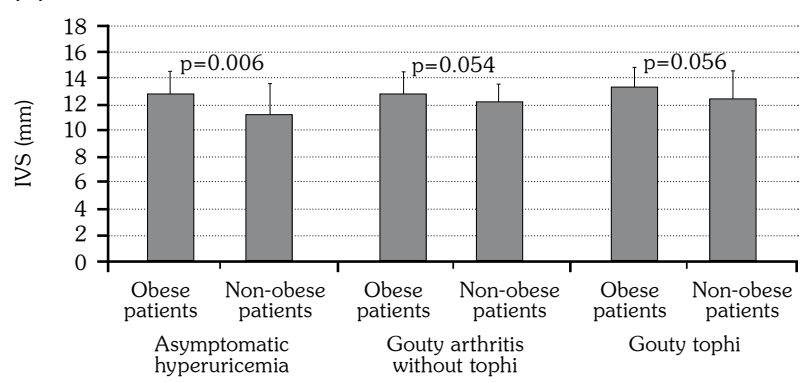

(c)

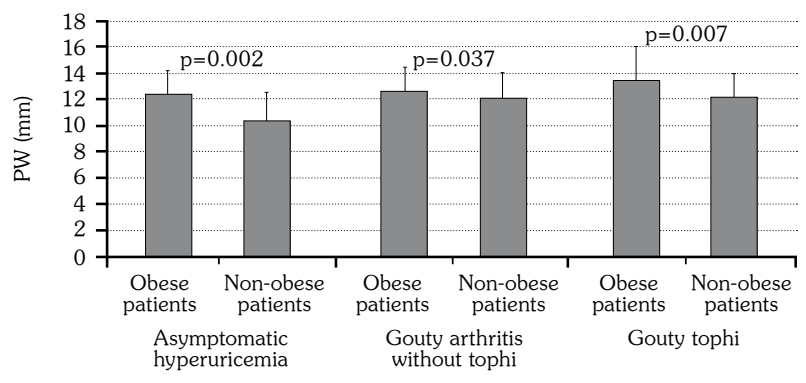

Figure 2. Mean and standard deviation of (a) left atrium size, (b) interventricular septum thickness and (c) thickness of posterior wall of left ventricle in obese and non-obese patients in three examined groups. LA: Left atrium; IVS: Interventricular septum; PW: Posterior wall. morphological heart changes compared to non-obese individuals despite the similar systolic and diastolic functional parameters and the comparable results for the FRS, accountable for a cardiovascular risk.

In general, obesity is characterized with increased blood volume, hemodynamic overload and hyperdynamic circulation. ${ }^{19}$ This in part could explain our observations of larger LA and thicker left ventricular PW in obese subjects versus non-obese in the three disease gradations. In the whole cohort, without considering the role of obesity, a lack of a clear deviation was found in the IVS in the course of the disease. PW thickness was numerically the highest in gouty tophi. We measured the largest size of LA under the most severe gout the tophaceous gout, but paradoxically, it was commensurable with those in asymptomatic hyperuricemia. In our case, we sought the explanation for this result in the equal prevalence of CKF in these patient groups. The literature supports the well-known LA enlargement in chronic kidney disease patients, including those with mild to moderate $\mathrm{CKF}^{28}$ In such cases, LA enlargement is mainly due to volume expansion and also to inflammation. ${ }^{29}$ Namely, inflammation is the other non-hemodynamic marker that correlates with LA size. ${ }^{30}$ There are now data in patients with gout, which make evident that the existence of subcutaneous tophi has a significant impact on LA enlargement. They suggest that maintenance of chronic inflammatory process and higher level of oxidative stress, in addition to hyperuricemia, are responsible for the alteration. ${ }^{10}$ Moreover, the severe inflammation and higher total body urate load in gouty tophi stage have been shown to be associated with higher risk

Table 3. Results from multiple linear regression, adjusted for age, with dependent variable posterior wall of left ventricle and an independent factor-body mass index

\begin{tabular}{|c|c|c|c|c|c|}
\hline Dependent variable - PW & Factors & Coefficient & $\mathrm{t}$ & $p$ & $\mathrm{R}^{2}$ \\
\hline \multirow{3}{*}{ Asymptomatic hyperuricemia } & Age & 0.05 & 3.22 & 0.002 & \multirow{3}{*}{0.319} \\
\hline & Body mass index & 0.16 & 4.07 & $<0.001$ & \\
\hline & Constant & 3.62 & 2.23 & 0.030 & \\
\hline \multirow{2}{*}{ Gouty arthritis without tophi } & Body mass index & 0.11 & 2.94 & 0.004 & \multirow{2}{*}{0.093} \\
\hline & Constant & 9.12 & 8.13 & $<0.001$ & \\
\hline \multirow{2}{*}{ Gouty tophi } & Body mass index & 0.11 & 2.09 & 0.041 & \multirow{2}{*}{0.068} \\
\hline & Constant & 9.39 & 5.84 & $<0.001$ & \\
\hline
\end{tabular}


of cardiovascular death. ${ }^{11}$ By registering the highest serum CRP in gouty tophi, despite the equality of the subjects with flare in both gout subdivisions, we indirectly judged for the highest level of persistent inflammation. Also, in recent years, the proinflammatory activity of the adipose tissue has been demonstrated in animal models due to its lipotoxic effect connected to cardiac steatosis and lipoapoptosis. ${ }^{31,32}$ We believe that the varying degrees of inflammation in the stages of gout along with the proinflammatory activity due to increased fat accumulation ${ }^{33}$ potentiate the detrimental effect on the heart leading to pronounced morphological heart changes. In this study, we focused on the role of obesity on the ultrasonographic heart alterations, particularly in the context of gout. This was confirmed by the constructed regression models, which revealed that weight gain is an independent predictor of left ventricular wall changes. The increase of $\mathrm{BMI}$ influenced thickening of the PW of the left ventricle in the three disease stages. Nevertheless, its impact was the weakest in gouty tophi patients, in whom it should be suspected that the above mentioned factors would likely be more damaging for the heart.

Furthermore, evidence exists to suggest that obesity impacts the diastole, impairs left ventricular relaxation and alters left ventricular filling indexes. ${ }^{34,35}$ However, in obese individuals, left ventricular filling pressures are usually normal at rest, ${ }^{36}$ which could explain our observation for the comparable values of $\mathrm{E} / \mathrm{E}^{\prime}$ in obese and non-obese in the disease continuum. Across the disease spectrum, gouty tophi patients had the worst diastolic function as a whole, without registering a great variation in the functional diastolic parameters between obese and nonobese subjects. Based on these results, we assume that maintenance of inflammatory state in chronic tophaceous gout and all its consequences has a great importance in the pathogenesis of left ventricular diastolic dysfunction as the abnormal fat accumulation.

It has been shown that the extent of cardiac remodeling in obesity increases with its severity and duration. ${ }^{19}$ In obesity, cardiac output is often higher, but myocardial contractility is reduced, even if the ejection fraction is normal. ${ }^{37}$ Often, obese compared to normal weight subjects have reduced midwall left ventricular fractional shortening and lower S' measured by TDI. ${ }^{38,39}$ Nevertheless, in our cohort, FS and S did not change significantly between obese and nonobese individuals, as we did not estimate a great variation in their mean values. In this context, it should be born in mind that in obese individuals, the presence of subclinical systolic and diastolic dysfunction can be judged by larger LA and thicker PW of the left ventricle. This led us to suggest that compensatory mechanisms aiming to preserve functioning of the heart, despite the morphological alterations, take place in patients with obesity.

Several limitations of our study deserve mention. This was a single-center cross-sectional study and laboratory parameters were measured at a fixed point of time. Also, FRS was not calculated in all subjects due to incomplete data for lipid panel. Due to poor ultrasound window in a minority of obese patients, FS and some TDI parameters were not recorded. Furthermore, in asymptomatic hyperuricemia, no additional imaging methods for detection of silent monosodium urate crystals were performed. Moreover, echocardiographic examinations were performed by one researcher. With this in mind, we are between the few who have determined cardiovascular risk in obese and non-obese in the spectrum of gout using a most widely applicable scoring system and transthoracic echocardiography.

In conclusion, given the comparable systolic and diastolic function in obese and non-obese, the scores obtained by using the FRS enabled us to procure a quick and accurate orientation on the cardiovascular risk. Following the assessment by this prediction tool, we calculated higher cardiovascular risk estimates in gout patients than in asymptomatic hyperuricemia, while a difference depending on body weight from the earlier to later disease stages was not seen. We suggest that combined application of a scoring system with a non-invasive and harmless imaging method would be an appropriate approach for the assessment of cardiovascular risk in the individual stages of gout, in which the prevalence of obesity is high.

\section{Declaration of conflicting interests}

The authors declared no conflicts of interest with respect to the authorship and/or publication of this article. 


\section{Funding}

The authors received no financial support for the research and/or authorship of this article.

\section{REFERENCES}

1. Kopelman PG, Caterson ID, Stock MJ, Dietz WH. Clinical Obesity in Adults and Children in Adults and Children. Massachusetts: Blackwell Publishing; 2005.

2. Ng M, Fleming T, Robinson M, Thomson B, Graetz $\mathrm{N}$, Margono C, et al. Global, regional, and national prevalence of overweight and obesity in children and adults during 1980-2013: a systematic analysis for the Global Burden of Disease Study 2013. Lancet 2014;384:766-81.

3. Poirier P, Giles TD, Bray GA, Hong Y, Stern JS, Pi-Sunyer FX, et al. Obesity and cardiovascular disease: pathophysiology, evaluation, and effect of weight loss: an update of the 1997 American Heart Association Scientific Statement on Obesity and Heart Disease from the Obesity Committee of the Council on Nutrition, Physical Activity, and Metabolism. Circulation 2006;113:898-918.

4. Choi HK, Atkinson K, Karlson EW, Curhan G. Obesity, weight change, hypertension, diuretic use, and risk of gout in men: the health professionals follow-up study. Arch Intern Med 2005;165:742-8.

5. Enomoto A, Kimura H, Chairoungdua A, Shigeta Y, Jutabha $\mathrm{P}$, Cha $\mathrm{SH}$, et al. Molecular identification of a renal urate anion exchanger that regulates blood urate levels. Nature 2002;417:447-52.

6. Juraschek SP, Miller ER, Gelber AC. Body mass index, obesity, and prevalent gout in the United States in 1988-1994 and 2007-2010. Arthritis Care Res (Hoboken) 2013;65:127-32.

7. Campion EW, Glynn RJ, DeLabry LO. Asymptomatic hyperuricemia. Risks and consequences in the Normative Aging Study. Am J Med 1987;82:421-6.

8. Roubenoff R, Klag MJ, Mead LA, Liang KY, Seidler $\mathrm{AJ}$, Hochberg MC. Incidence and risk factors for gout in white men. JAMA 1991;266:3004-7.

9. Chen SY, Chen CL, Shen ML. Severity of gouty arthritis is associated with Q-wave myocardial infarction: a large-scale, cross-sectional study. Clin Rheumatol 2007;26:308-13.

10. Pan KL, Lin JC, Lin CL, Chen MC, Chang PJ, Hsiao $\mathrm{JF}$, et al. The effects of gout on left atrial volume remodelling: a prospective echocardiographic study. Rheumatology (Oxford) 2014;53:867-74.

11. Perez-Ruiz F, Martínez-Indart L, Carmona L, HerreroBeites AM, Pijoan JI, Krishnan E. Tophaceous gout and high level of hyperuricaemia are both associated with increased risk of mortality in patients with gout. Ann Rheum Dis 2014;73:177-82.

12. Bombelli M, Facchetti R, Cuspidi C, Villa P, Dozio D, Brambilla $G$, et al. Prognostic significance of left atrial enlargement in a general population: results of the PAMELA study. Hypertension 2014;64:1205-11.

13. Correale M, Totaro A, leva R, Ferraretti A, Musaico F, Di Biase M. Tissue Doppler imaging in coronary artery diseases and heart failure. Curr Cardiol Rev 2012;8:43-53.

14. Yu CM, Sanderson JE, Marwick TH, Oh JK. Tissue Doppler imaging a new prognosticator for cardiovascular diseases. J Am Coll Cardiol 2007;49:1903-14.

15. Verdecchia P, Reboldi G, Schillaci G, Borgioni C, Ciucci A, Telera MP, et al. Value of a simple echocardiographic linear predictor of left ventricular mass in systemic hypertension. Am $\mathrm{J}$ Cardiol 1999;84:1209-14.

16. Galderisi M, Henein MY, D'hooge J, Sicari R, Badano LP, Zamorano JL, et al. Recommendations of the European Association of Echocardiography: how to use echo-Doppler in clinical trials: different modalities for different purposes. Eur $\mathrm{J}$ Echocardiogr 2011;12:339-53.

17. Mogelvang R, Sogaard P, Pedersen SA, Olsen NT, Marott JL, Schnohr P, et al. Cardiac dysfunction assessed by echocardiographic tissue Doppler imaging is an independent predictor of mortality in the general population. Circulation 2009;119:2679-85.

18. Lang RM, Bierig M, Devereux RB, Flachskampf FA, Foster E, Pellikka PA, et al. Recommendations for chamber quantification. Eur $\mathrm{J}$ Echocardiogr 2006;7:79-108.

19. Vasan RS. Cardiac function and obesity. Heart 2003;89:1127-9.

20. Neogi T, Jansen TL, Dalbeth N, Fransen J, Schumacher HR, Berendsen D, et al. 2015 Gout Classification Criteria: an American College of Rheumatology/European League Against Rheumatism collaborative initiative. Arthritis Rheumatol 2015;67:2557-68.

21. Clinical guidelines on the identification, evaluation, and treatment of overweight and obesity in adults: executive summary. Expert Panel on the Identification, Evaluation, and Treatment of Overweight in Adults. Am J Clin Nutr 1998;68:899-917.

22. Expert Panel on Detection, Evaluation, and Treatment of High Blood Cholesterol in Adults. Executive Summary of The Third Report of The National Cholesterol Education Program (NCEP) Expert Panel on Detection, Evaluation, And Treatment of High Blood Cholesterol In Adults (Adult Treatment Panel III). JAMA 2001;285:2486-97.

23. Grundy SM, Cleeman JI, Merz CN, Brewer HB Jr, Clark LT, Hunninghake DB, et al. Implications of recent clinical trials for the National Cholesterol Education Program Adult Treatment Panel III guidelines. Circulation 2004;110:227-39.

24. Kopple JD. National kidney foundation K/DOQI clinical practice guidelines for nutrition in chronic renal failure. Am J Kidney Dis 2001;37:66-70. 
25. Heine GH, Gerhart MK, Ulrich C, Köhler H, Girndt M. Renal Doppler resistance indices are associated with systemic atherosclerosis in kidney transplant recipients. Kidney Int 2005;68:878-85.

26. Quiñones MA, Otto CM, Stoddard M, Waggoner A, Zoghbi WA. Recommendations for quantification of Doppler echocardiography: a report from the Doppler Quantification Task Force of the Nomenclature and Standards Committee of the American Society of Echocardiography. J Am Soc Echocardiogr 2002;15:167-84.

27. Chahal NS, Lim TK, Jain P, Chambers JC, Kooner JS, Senior R. Normative reference values for the tissue Doppler imaging parameters of left ventricular function: a population-based study. Eur J Echocardiogr 2010;11:51-6.

28. Paoletti E, Zoccali C. A look at the upper heart chamber: the left atrium in chronic kidney disease. Nephrol Dial Transplant 2014;29:1847-53.

29. Essig M, Escoubet B, de Zuttere D, Blanchet F, Arnoult F, Dupuis E, et al. Cardiovascular remodelling and extracellular fluid excess in early stages of chronic kidney disease. Nephrol Dial Transplant 2008;23:239-48.

30. Rao AK, Djamali A, Korcarz CE, Aeschlimann $\mathrm{SE}$, Wolff MR, Stein JH. Left atrial volume is associated with inflammation and atherosclerosis in patients with kidney disease. Echocardiography 2008;25:264-9.

31. Zhou YT, Grayburn P, Karim A, Shimabukuro M, Higa M, Baetens D, et al. Lipotoxic heart disease in obese rats: implications for human obesity. Proc Natl Acad Sci U S A 2000;97:1784-9.
32. Chiu HC, Kovacs A, Ford DA, Hsu FF, Garcia R, Herrero $P$, et al. A novel mouse model of lipotoxic cardiomyopathy. J Clin Invest 2001;107:813-22.

33. Lyon CJ, Law RE, Hsueh WA. Minireview: adiposity, inflammation, and atherogenesis. Endocrinology 2003;144:2195-200.

34. Morricone L, Malavazos AE, Coman C, Donati C, Hassan T, Caviezel F. Echocardiographic abnormalities in normotensive obese patients: relationship with visceral fat. Obes Res 2002;10:489-98.

35. Pascual M, Pascual DA, Soria F, Vicente T, Hernández AM, Tébar FJ, et al. Effects of isolated obesity on systolic and diastolic left ventricular function. Heart 2003;89:1152-6.

36. Wong CY, O'Moore-Sullivan T, Leano R, Byrne N, Beller E, Marwick TH. Alterations of left ventricular myocardial characteristics associated with obesity. Circulation 2004;110:3081-7.

37. Garavaglia GE, Messerli FH, Nunez BD, Schmieder RE, Grossman E. Myocardial contractility and left ventricular function in obese patients with essential hypertension. Am J Cardiol 1988;62:594-7.

38. Aurigemma GP, Silver KH, Priest MA, Gaasch WH. Geometric changes allow normal ejection fraction despite depressed myocardial shortening in hypertensive left ventricular hypertrophy. J Am Coll Cardiol 1995;26:195-202.

39. Avelar E, Cloward TV, Walker JM, Farney RJ, Strong $\mathrm{M}$, Pendleton RC, et al. Left ventricular hypertrophy in severe obesity: interactions among blood pressure, nocturnal hypoxemia, and body mass. Hypertension 2007;49:34-9. 